\title{
PAIR-PRODUCTION AVALANCHES REVISITED
}

\author{
F. Curtis Michel \\ Space Physics and Astronomy Department, Rice University
}

As noted previously in this meeting, any millisecond pulsar that is not perfectly aligned must $o b$ viously be strongly charged electrostatically simply because the wave zone is so close to the surface that any trapped neutralizing electrosphere over the polar caps will be driven away beyond 6 or 7 neutronstar radii. Acceleration of stray charges (e.g., from the ISM or a nearby orbiting disk) in such a highly charged system automatically produces coherently radiating bunches of downward moving particles (Michel 1990), this coherent radiation simply reflecting from the surface (or missing the star entirely, as an alternative possibility). This mechanism, suggestive of how pulsars might operate, we will call a pair-production avalanche.

The discovery that the non-neutral nature of plasmas about neutron stars leads to space-charge trapping (and therefore "quiet pulsars": cf. Michel (1980), Krause-Polstorff and Michel (1985a, b)), has resulted in a number of suggestions that have been forwarded in attempts to resuscitate the Goldreich and Julian (1969) model which assumed no such trapping. For example, Cheng (1985) has proposed that dust grains might provide the the additional plasma to fill the magnetosphere, the idea being to return to a filled magnetosphere where corotation and centrifugal forces would force a plasma wind and presumably pulsar action. Earlier, Rylov (1984), Mestel et al. (1985), and Ruderman (1985) have proposed that the interstellar medium might enter the pulsar system, but this is difficult given the expected generation of large amplitude electromagnetic waves by a non-axisymmetric system (Arons and Barnard 1983, Michel 1987a), which act to sweep out plasma. Instead, we follow up on the diametrically opposite view that the system is never filled but remains highly charged.

The idea of a pair-production avalanche is not new; it is the older pair-production cascade idea (Sturrock 1971), but operating in a different regime (some authors have used the two terms "cascade" and "avalanche" interchangeably). Previously one imagined that particles were accelerated to large Lorentz factors close to the neutron star and later, as they coasted outward, they might emit some $\gamma$ rays that magnetically pair-produce less-energetic pairs, a cascade that quickly terminated. When the particles are radiation-reaction limited, the cascade does not die out, but rather their numbers exponentiate because the "secondaries" almost immediately become new "primaries". Furthermore, because the electrons have large Lorentz factors and it is only a short distance to the neutron star, the $\gamma$-rays do not propagate any significant distance ahead of the emitting electron. Thus all of the secondary, tertiary, etc., electrons are essentially created as companions of the primary electron and therefore automatically form together in a sheet-like (or ribbonlike) bunch as illustrated in figure 1.

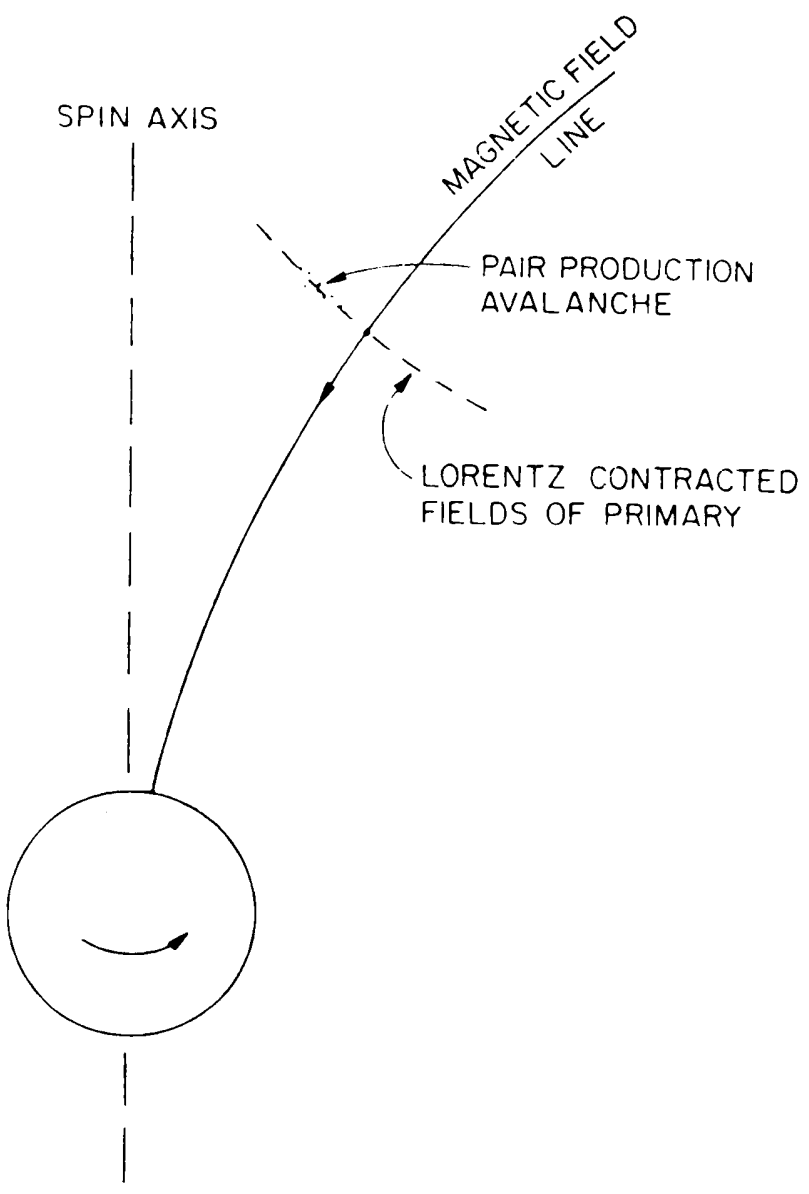

Figure 1

At first an electron introduced into a charged neutron-star system (here we consider a typical 1s pulsar and $10^{12}$-Gauss magnetic field) is simply accelerated but quickly becomes radiation-reaction limited with a Lorentz factor of order $5 \times 10^{7}$, radiating $\gamma$-rays with an equivalent Lorentz factor (photon energy divided by $m c^{2}$ ) of about $10^{4}$. As the electron advances, the magnetic field increases to the point (roughly 27 radii) where pair production by these $\gamma$-rays first becomes possible, taking into account that both the electron and $\gamma$-ray energies have increased owing to the increasing acceleration 
toward the star. No pair production actually occurs because the $\gamma$-rays are initially propagating parallel to the magnetic field lines, but by 13.6 radii these harder $\gamma$-rays are crossing even stronger fields at significant angles and pair production begins in earnest. Each electron produced has an energy comparable to the initial $\gamma$-ray (three to four orders of magnitude weaker than that of the primary particle) but because that energy is radiation-reaction limited, each new electron is almost immediately accelerated up to the same energy as the primary (and the positrons are halted and then ejected from the system because once they reach the light cylinder they would be ejected even if they had no energy, while in fact they should be quite energetic).

To estimate the dimensions of the secondary electron bunch, we use the lateral distance that the $\gamma$-rays must depart from the original magnetic field line before they pair-produce. This production takes place on magnetic field lines poleward of the primary electron and essentially along side of the primary. Rather than thinking of the $\gamma$-rays as running ahead of the electron before they pairproduce, one can take the view that the electric field of the electron is Lorentz-contracted into a comoving sheet and, as the electron moves along the curved magnetic field lines, the sheet on the outside of the turn lags (i.e., is radiated). It is in this lagging portion that the pairs are created. The multiplication factor is quite large because a new pair is formed about every meter at first. However, the bunch size does not exponentiate quite that rapidly because the $\gamma$-rays from the new particles do not immediately pair-produce. Because the magnetic field is rapidly increasing in strength, the curvature radius is decreasing, and the $\gamma$-ray energies are increasing; $\gamma$-rays from the steadily growing cloud of secondaries begin to produce tertiary electrons at around 10 radii, followed by additional stages at $8.5,7.7$, and 7.0 radii. All of these new particles are essentially sitting slightly further out in a growing intense electromagnetic pulse. As the neutron star is approached, the bunch size is shrinking to dimensions of order meters owing to the magnetic field line packing, while the total number of electrons is essentially climbing exponentially. The amount of energy going into coherent radiation is finally becoming significant.

This runaway increase ceases, however, when another effect takes place: positron trapping by the highly charged bunch, which occurs when the electric field of the bunch becomes comparable to the accelerating field of the star, which in the model takes place at about 6.6 stellar radii. Now the net charge (here of order $10^{20}$ electrons) of the bunch cannot increase further, although further pair production may increase the total number of particles. Regardless, the coherent radiation is now roughly a fixed factor (about $10^{-5}$ ) of the available energy, which is, in turn, mainly emitted close to the surface. Note that positron trapping is largely a modelindependent limitation if bunching is due to pairproduction in the first place. Moreover, the downward moving bunch is trailing positrons that are accelerated out of the system, which is the current closure mechanism. Thus the closed currents and the coherent radiation result from one and the same mechanism.

Sturrock (1971) had an interestingly similar picture, but because he imagined the primary electrons to be accelerated out from the star, he thought that the secondary positrons would be counterstreaming through these electrons, leading to a twostream instability. He then assumed the electrons to be bunched into sheets by this instability, and estimated the surface concentration of electrons on such sheets by assuming they produced a local electrostatic field comparable to the accelerating field (by "a mechanism which we do not investigate"). In our model, this mechanism is just the above positrontrapping. Having assumed this bunching to occur, Sturrock proposed this trapping as a vehicle for the bunched sheet of electrons to "shadow" any pairs produced and allow them to be ejected from the system by trailing the sheet itself. In our model, such trailing is the mechanism that limits the total sheet charge and hence coherence factor of the bunch. In a more recent paper on cascades as a model for $\gamma$-ray bursts (Sturrock, Harding, and Daugherty 1989), the possibility that particles might be accelerated to radiation-reaction limited energy is mentioned in connection with disk/neutron-star models (Michel 1985, Epstein 1985), but not explored further.

I have taken my allotted time, but I would like to emphasize what a difference the paradigm shift can make in going from the almost universal fixation on radiation from particles moving outward to consideration of radiation from particles moving inward. Now, the previous difficulties with curvature radiation as a radio emission mechanism vanish: The bunch is formed in situ rather than accumulated from ambient particles (the bunching rate problem); the velocity dispersion within the bunch is irrelevant because the particles are highly relativistic and have only a short distance to travel (the bunch dissipation problem); and the bunch is geometrically constrained to be nearly orthogonal to the magnetic field direction (the tilt problem, as discussed elsewhere in this conference by Melrose). 